\title{
SPECTRAL AND FREDHOLM PROPERTIES OF OPERATORS IN ELEMENTARY NEST ALGEBRAS
}

\author{
BRUCE A. BARNES AND JON M. CLAUSS
}

\begin{abstract}
Some spectral and Fredholm properties are proved for linear operators which leave invariant certain nests of closed subspaces.
\end{abstract}

\section{INTRODUCTION}

Throughout, $X$ is an infinite-dimensional Banach space, $B(X)$ is the algebra of all bounded linear operators on $X$, and for $T \in B(X), \sigma(T)$ is the spectrum of $T$. For $T \in B(X), \operatorname{Lat}(T)$ is the collection of all closed subspaces $M$ of $X$ such that $T(M) \subseteq M$. When $\operatorname{Lat}(T)$ contains certain types of nests (totally ordered collections), then this may affect the spectral or Fredholm properties of $T$. A famous example is due to J. Ringrose: When $T$ is a compact operator and $\operatorname{Lat}(T)$ contains a continuous nest, then $\sigma(T)=\{0\}$ [3, Corollary 4.3.11].

In this paper we consider the spectral and Fredholm theory of operators $T$ such that $\operatorname{Lat}(T)$ contains an elementary nest of one of the two types described below.

Definition 1. A collection $\mathscr{E}$ of closed subspaces of $X$ is an elementary upper nest (EUN) if $\mathscr{E}=\left\{M_{0}: 0 \leq n \leq \infty\right\}$, where

(i) $M_{0}=\{0\}, M_{\infty}=X$, and $M_{n} \subseteq M_{n+1}$ for $n \geq 0$;

(ii) $M_{n}$ is f.d. (finite-dimensional) for $0 \leq n<\infty$;

(iii) $\bigcup\left\{M_{n}: 0 \leq n<\infty\right\}$ is dense in $X$.

The collection $\mathscr{E}$ as above is an elementary lower nest (ELN) if

(i) $M_{\infty}=\{0\}, M_{0}=X$, and $M_{n+1} \subseteq M_{n}$ for $n \geq 0$;

(ii) $M_{n} / M_{n+1}$ is f.d. for $n \geq 0$;

(iii) $\bigcap\left\{M_{n}: 0 \leq n<\infty\right\}=\{0\}$.

As an example, assume $\left\{X_{k}: k \geq 1\right\}$ is a linearly independent collection of f.d. subspaces of $X$ such that $X=\bigoplus \sum_{k=1}^{\infty} X_{k}$ (for each $x \in X$, there exists a unique sequence $\left\{x_{k}\right\}_{k \geq 1}$ with $x_{k} \in X_{k}, k \geq 1$, and $\left.x=\sum_{k=1}^{\infty} x_{k}\right)$. Setting $M_{0}=\{0\}, M_{\infty}=X$, and $M_{n}=\sum_{k=1}^{n} X_{k}$, we have $\mathscr{E}=\left\{M_{n}: 0 \leq n \leq \infty\right\}$ is an EUN. An operator $S \in B(X)$ such that $S\left(X_{n}\right) \subseteq X_{n-1}$ for $n \geq 1 \quad\left(X_{0}=\{0\}\right)$ is a type of backward shift operator. Clearly, $\mathscr{E} \subseteq \operatorname{Lat}(S)$. Results in this paper

Received by the editors October 27, 1993; originally communicated to the Proceedings of the $A M S$ by Palle E. T. Jorgensen.

1991 Mathematics Subject Classification. Primary 47A10, 47A15, 47B30.

Key words and phrases. Elementary nest, spectrum, Fredholm operator, index. 
show that $\sigma(S)$ is connected [Theorem 6], and that if $\lambda-S$ is Fredholm on $X$, then $\operatorname{ind}(\lambda-S) \leq 0$ [Corollary 8]. Here ind $(T)$ denotes the usual index of a Fredholm operator $T \in B(X)$.

Also, note in this same situation that if $N_{0}=X, N_{\infty}=\{0\}$, and $N_{n}=\{x=$ $\left.\sum_{k=1}^{\infty} x_{k} \in X: x_{k}=0,1 \leq k \leq n\right\}, 1 \leq n<\infty$, then $\mathscr{N}=\left\{N_{n}: 0 \leq n \leq \infty\right\}$ is an ELN.

\section{SPECTRAL PROPERTIES}

Throughout $T$ is an operator in $B(X)$. The object of this section is to show that when $\mathscr{E}$ is an EUN or an ELN, and $\mathscr{E} \subseteq \operatorname{Lat}(T)$, then this affects the spectral properties of $T$. Let $\operatorname{Alg}(\mathscr{E})=\{S \in B(X): \mathscr{E} \subseteq \operatorname{Lat}(S)\}$. The algebra $\operatorname{Alg}(\mathscr{E})$ is a closed subalgebra of $B(X)$ which contains the identity operator. Properties of compact operators in $\operatorname{Alg}(\mathscr{N})$, where $\mathscr{N}$ is an elementary nest, are studied in [2].

Proposition 2. When $\mathscr{E}$ is either an $E U N$ or an $E L N$, then $A=\operatorname{Alg}(\mathscr{E})$ is inverse closed in $B(X)$ (that is, if $T \in A$ and $T^{-1} \in B(X)$, then $T^{-1} \in A$ ).

Proof. Assume $\mathscr{E}=\left\{M_{n}: 0 \leq n \leq \infty\right\}$. It suffices in either case to show that if $T \in A$ and $T^{-1} \in B(X)$, then $T\left(M_{n}\right)=M_{n}$ for all $n$. When $\mathscr{E}$ is an EUN, then this is obvious since $T$ is $1-1$ on $M_{n}$ and $M_{n}$ is f.d., so $T\left(M_{n}\right)=M_{n}$. Now suppose $\mathscr{E}$ is an ELN. We do this case by induction. Certainly $T\left(M_{0}\right)=M_{0}$. Suppose $T\left(M_{n}\right)=M_{n}$ for some $n$. This implies $T$ maps $M_{n} / M_{n+1}$ onto $M_{n} / M_{n+1}$. Therefore $T$ is $1-1$ on $M_{n} / M_{n+1}$. Fix $z \in M_{n+1}$. By the induction hypothesis $\exists w \in M_{n}$ such that $T w=z$. Thus, $T\left(w+M_{n+1}\right)=z+M_{n+1}$. Since $T$ is $1-1$ on $M_{n} / M_{n+1}, w \in M_{n+1}$.

Next we prove a key result. For $T \in B(X)$, let $\mathscr{N}(T)$ be the null space of $T$ and $\mathscr{R}(T)$ be the range of $T$.

Theorem 3. Assume $\mathscr{E} \subseteq \operatorname{Lat}(T)$.

(1) If $\mathscr{E}$ is EUN and $T$ is surjective on $M_{n+1} / M_{n}$ for $n \geq 0$, then $\mathscr{R}(T)$ is dense in $X$.

(2) If $\mathscr{E}$ is an ELN and $T$ is injective on $M_{n} / M_{n+1}$ for $n \geq 0$, then $\mathscr{N}(T)=$ $\{0\}$.

(3) If $\mathscr{E}$ is an $E L N$ and $\mathscr{N}(T) \neq\{0\}$, then $\mathscr{R}(T)$ is not dense in $X$.

Proof. Assume $T$ is as in (1). Since $T$ is surjective on $M_{1} / M_{0}, M_{0}=\{0\}$, then $T\left(M_{1}\right)=M_{1}$. We verify by induction that $M_{n}=T\left(M_{n}\right)$ for all $n \geq 1$. Assume $T\left(M_{n}\right)=M_{n}$. By hypothesis $T\left(M_{n+1} / M_{n}\right)=M_{n+1} / M_{n}$. Assume $z \in M_{n+1}$. Then $\exists w \in M_{n+1}$ such that $z-T(w) \in M_{n}$. By the induction hypothesis, $\exists x \in M_{n}$ with $T(x)=z-T(w)$. Thus $x+w \in M_{n+1}$ and $T(x+w)=z$. Therefore, $T\left(M_{n+1}\right)=M_{n+1}$. It follows that $\bigcup_{n=1}^{\infty} M_{n} \subseteq \mathscr{R}(T)$, so $\mathscr{R}(T)$ is dense in $X$.

Now assume $T$ is as in (2). Suppose $y \in X, y \neq 0$, and $T(y)=0$. Choose $n$ such that $y \in M_{n}$ and $y \notin M_{n+1}$. Then $y+M_{n+1} \in M_{n} / M_{n+1}, y+M_{n+1} \neq$ $0+M_{n+1}$. Also $T\left(y+M_{n+1}\right)=T(y)+M_{n+1}=0+M_{n+1}$, a contradiction.

Assume that $T$ is as in (3), so $\mathscr{N}(T) \neq\{0\}$. By (2), $\exists n$ such that $T$ is not 1-1 on $M_{n} / M_{n+1}$. Since $M_{n} / M_{n+1}$ is f.d., $T\left(M_{n} / M_{n+1}\right)$ is a proper subspace of $M_{n} / M_{n+1}$. This implies $\exists Z$ an f.d. subspace such that $T\left(M_{n}\right) \subseteq Z \oplus M_{n+1} \neq$ $M_{n}$. Now $X=Y \oplus M_{n}$, where $Y$ is an f.d. subspace. $T(X) \subseteq T(\bar{Y})+T\left(M_{n}\right) \subseteq$ 
$T(Y)+Z+M_{n+1}$. This last subspace is a closed proper subspace of $X$, so (3) holds.

We use the following notation for various parts of the spectrum of an operator $T \in B(X)$ :

$$
\begin{gathered}
\sigma_{p}(T) \equiv \text { the point spectrum (eigenvalues) of } T \\
\begin{aligned}
\sigma_{c}(T) & \equiv \text { the continuous spectrum of } T \\
& \equiv\left\{\lambda \notin \sigma_{p}(T): \overline{\mathscr{R}(\lambda-T)}=X, \mathscr{R}(\lambda-T) \neq X\right\}
\end{aligned} \\
\sigma_{r}(T) \equiv \text { the residual spectrum of } T \equiv\left\{\lambda \notin \sigma_{p}(T): \overline{\mathscr{R}(\lambda-T)} \neq X\right\} .
\end{gathered}
$$

Corollary 4. Assume $\mathscr{E} \subseteq \operatorname{Lat}(T)$.

(1) If $\mathscr{E}$ is an EUN, then $\sigma(T)=\sigma_{p}(T) \cup \sigma_{c}(T)$.

(2) If $\mathscr{E}$ is an $E L N$, and $T\left(M_{n}\right) \subseteq M_{n+1}$ for $n \geq 0$, then $\sigma_{p}(T) \backslash\{0\}$ is empty.

Proof. (1) It suffices to show that $\sigma_{r}(T)$ is empty. Suppose not, so $\exists \lambda$ with $\mathscr{N}(\lambda-T)=\{0\}$ and $\mathscr{R}(\lambda-T)$ not dense in $X$. By Theorem $3(1), \lambda-T$ is not surjective on $M_{n+1} / M_{n}$ for some $n$. This implies that $\lambda-T$ is not surjective on the f.d. space $M_{n+1}$. Therefore, $\lambda-T$ is not 1-1 on $M_{n+1}$. Thus, $\mathscr{N}(\lambda-T) \neq\{0\}$, a contradiction.

(2) Assume $\lambda \neq 0,(\lambda-T) x=0$, and $x \neq 0$. Since $\bigcap\left\{M_{k}: 0 \leq k<\infty\right\}=$ $\{0\}, \exists n$ such that $x \notin M_{n+1}$. We may assume $n$ is the smallest nonnegative integer with this property, so $x \in M_{n}$ and $x \notin M_{n+1}$. But $\lambda x=T x \in M_{n+1}$ by hypothesis. Thus $x \in M_{n+1}$, a contradiction.

Assume $J$ is a proper closed ideal in a Banach algebra $A$ with identity. It is a fact, from the holomorphic operational calculus, that when $T \in J$ and $f$ is holomorphic on some open neighborhood of $\sigma(T)$ and $f(0)=0$, then $f(T) \in J$. We apply this to the situation when $T \in J$ has disconnected spectrum.

Assertion 5. When $T \in J$ has disconnected spectrum in $A$, then some nonzero spectral idempotent of $T$ is in $J$.

To verify this, suppose $\sigma_{A}(T)=\Delta \cup \Gamma$, where $\Delta$ and $\Gamma$ are both nonempty open and closed subsets of $\sigma_{A}(T)$ and $\Delta$ and $\Gamma$ are disjoint. Assume $0 \notin \Delta$. Choose disjoint open subsets $U$ and $V$ of the complex plane such that $\Delta \subseteq U$ and $\Gamma \cup\{0\} \subseteq V$. Let $f \equiv 1$ on $U, f \equiv 0$ on $V$. Then, as remarked above, $F(T) \in J$. Thus Assertion 5 holds.

Theorem 6. Assume $\mathscr{E}=\left\{M_{k}: 0 \leq k \leq \infty\right\} \subseteq \operatorname{Lat}(T)$.

(1) If $\mathscr{E}$ is an EUN and $T\left(M_{n}\right) \subseteq M_{n-1}$ for $1 \leq n<\infty$, then $\sigma(T)$ is connected.

(2) If $\mathscr{E}$ is an ELN and $T\left(M_{n-1}\right) \subseteq M_{n}$ for $1 \leq n<\infty$, then $\sigma(T)$ is connected.

Proof. Let $A=\operatorname{Alg}(\mathscr{E})$. When $\mathscr{E}$ and $T$ are as in (1), let

$$
J=\left\{S \in A: S\left(M_{n}\right) \subseteq M_{n-1} \text { for } 1 \leq n<\infty\right\} .
$$

When $\mathscr{E}$ and $T$ are as in (2), let

$$
J=\left\{S \in A: S\left(M_{n-1}\right) \subseteq M_{n} \text { for } 1 \leq n<\infty\right\} .
$$


In both cases $J$ is a proper closed ideal of $A$ and $T \in J$. We claim that in both cases $J$ contains no nonzero projection. Once this fact is established, the theorem follows from Assertion 5 above.

Assume $E=E^{2} \in J$. Let $J$ be as in (3), and $\mathscr{E}$ an EUN. If $E \neq 0$, then $E\left(M_{n}\right) \neq\{0\}$ for some $n$. Therefore $\exists x \in M_{n}, x \neq 0$, with $x=E x$. Then $x=E x \in M_{n-1}$ since $E \in J$. Repeating this argument $n$ times, we have $x=E x \in M_{0}=\{0\}$, a contradiction.

Now assume $J$ is as in (4), and $\mathscr{E}$ is an ELN. If $E \neq 0, \exists x \in M_{0}=X, x \neq$ 0 , with $x=E x$. Suppose $x \in M_{n}$. Then $x=E x \in M_{n+1}$. Thus by induction it follows that $x \in M_{n}$ for all $n$. Therefore $x \in \bigcap\left\{M_{n}: 0 \leq n<\infty\right\}=\{0\}$, a contradiction.

Theorem 6 applies to shift-type and backward shift-type operators. In fact, the spectrum of these operators is often a disk centered at 0 . We illustrate this with an example. Assume that $X=\bigoplus \sum_{k=1}^{\infty} X_{k}$, where each $X_{k}$ is f.d. (as in the Introduction). For each $\mathscr{\odot} \in \mathbb{R}$, assume that the operator $V_{\mathscr{Q}}$ defined below is everywhere defined and bounded on $X$ : For $x \in X, x=\sum_{k=1}^{\infty} x_{k}, x_{k} \in X_{k}$,

$$
V_{\mathscr{O}}(x)=\sum_{k=1}^{\infty} e^{i k \mathscr{O}} x_{k}
$$

Then clearly $V_{(-\mathscr{O})}=V_{\mathscr{\theta}}^{-1}$ for all $\mathscr{O} \in \mathbb{R}$. Now assume $S \in B(X)$ and $S\left(X_{n}\right) \subseteq$ $X_{n-1}$ for $n \geq 1$. ( $S$ is a backward shift-type operator.) Fix $x \in X, x=$ $\sum_{k=1}^{\infty} x_{k}, x_{k} \in X_{k}$, and let $y_{k-1}=S\left(x_{k}\right) \in X_{k-1} \quad$ (as before, $\left.y_{0}=0\right)$. Then

$$
V_{\theta}^{-1} S V_{\Theta} x=V_{\theta}^{-1} S \sum_{k=1}^{\infty} e^{i k \theta} x_{k}=V_{\Theta}^{-1} \sum_{k=2}^{\infty} e^{i k \theta} y_{k-1}=e^{i \theta}\left(\sum_{k=2}^{\infty} y_{k-1}\right)=e^{i \theta} S x \text {. }
$$

Thus, $e^{i \theta} S$ is similar to $S$, and therefore

$$
\sigma(S)=e^{i \theta} \sigma(S) \text { for all } \mathscr{O} \in \mathbb{R} \text {. }
$$

This means that when $\lambda \in \sigma(S)$, the circle $\left\{e^{i \mathscr{\theta}} \lambda: \mathscr{O} \in \mathbb{R}\right\} \subseteq \sigma(S)$. Since $0 \in \sigma(S)$ and $\sigma(S)$ is connected [Theorem 6], it follows that $\sigma(S)$ is a disk centered at 0 . Results of this type are well known for certain shifts and backward shifts; see [5] for example.

\section{FREDHOLM PROPERTIES}

In this section we consider the Fredholm properties of an operator $T$ when $\mathscr{E}$ is an EUN or an ELN and $\mathscr{E} \subseteq \operatorname{Lat}(T)$. First we establish a basic perturbation result.

When $A=\operatorname{Lat}(\mathscr{E})$, we set $\mathscr{F}(A) \equiv$ the space of all operators in $A$ which have f.d. range.

Proposition 7. Let $A=\mathrm{Alg}(\mathscr{E})$, and assume $T \in A$.

(1) If $\mathscr{E}$ is an EUN, then $\exists K \in \overline{\mathscr{F}(A)}$ such that $\mathscr{R}(T+K)$ is dense in $X$.

(2) If $\mathscr{E}$ is an $E L N$, then $\exists J \in \bar{F}(A)$ such that $\mathscr{N}(T+J)=\{0\}$.

Proof. First assume that $\mathscr{E}$ is an EUN. For each $n \geq 1$ choose an f.d. subspace $Y_{n}$ of $X$ such that $M_{n}=Y_{n} \oplus M_{n-1}$. Let $E_{n}$ be a projection in $B(X)$ such that $E_{n}\left(M_{n-1}\right)=\{0\}$ and $\mathscr{R}\left(E_{n}\right)=Y_{n}$. Then

$$
E_{n}\left(M_{j}\right)=\{0\} \text { for } 1 \leq j \leq n-1, \quad \text { and } E_{n}\left(M_{j}\right)=Y_{n} \subseteq M_{j} \text { for } n \leq j<\infty \text {. }
$$


It follows that $E_{n} \in \mathscr{F}(A)$. Now $E_{n}$ acts as the identity operator on $M_{n} / M_{n-1}$, and the spectrum of $T$ on $M_{n} / M_{n-1}$ is finite. Therefore, we can choose $\varepsilon_{n}>0$ such that $\varepsilon_{n}\left\|E_{n}\right\|<2^{-n}$ and $T+\varepsilon_{n} E_{n}$ is invertible on $M_{n} / M_{n-1}$. Set $K=$ $\sum_{n=1}^{\infty} \varepsilon_{n} E_{n} \in \bar{F}(A)$. By the construction $T+K$ is invertible on $M_{n} / M_{n-1}$ for $n \geq 1$. It follows from Theorem $3(1)$ that $\mathscr{R}(T+K)$ is dense in $X$.

Now assume $\mathscr{E}$ is an ELN. For $1 \leq n<\infty$, choose an f.d. subspace $Z_{n}$ such that $M_{n-1}=Z_{n} \oplus M_{n}$. Let $F_{n}$ be a projection in $B(X)$ with $F_{n}\left(M_{n}\right)=\{0\}$ and $\mathscr{R}\left(F_{n}\right)=Z_{n}$. Then

$$
F_{n}\left(M_{j}\right) \subseteq F_{n}\left(M_{n}\right)=\{0\} \text { for } n \leq j<\infty,
$$

and

$$
F_{n}\left(M_{j}\right)=Z_{n} \subseteq M_{j} \text { for } 1 \leq j<n .
$$

Therefore, $F_{n} \in \mathscr{F}(A)$. As before, choose $\varepsilon_{n}>0$ with $\varepsilon_{n}\left\|F_{n}\right\|<2^{-n}$ and $T+\varepsilon_{n} F_{n}$ invertible on $M_{n-1} / M_{n}, 1 \leq n<\infty$. Let $J=\sum_{n=1}^{\infty} \varepsilon_{n} F_{n} \in \overline{\mathscr{F}(A)}$. By the construction $T+J$ is invertible on $M_{n-1} / M_{n}$ for $1 \leq n<\infty$. Therefore, by Theorem $3(2), \mathcal{N}(T+J)=\{0\}$.

Let $\Phi(X)$ denote the set of Fredholm operators in $B(X)$. Also, let $\Phi^{0}(X)$ be the set of all $T \in \Phi(X)$ such that $\operatorname{ind}(T)=0 \quad(\operatorname{ind}(T) \equiv$ the index of $T)$.

Corollary 8. Assume $\mathscr{E} \subseteq \operatorname{Lat}(T)$ and $T \in \Phi(X)$.

(1) If $\mathscr{E}$ is an $E U N$, then ind $(T) \geq 0$.

(2) If $\mathscr{E}$ is an $E L N$, then $\operatorname{ind}(T) \leq 0$.

Proof. We prove (1) only (the proof of (2) is similar). Assume $\mathscr{E}$ is an EUN, $\mathscr{E} \subseteq \operatorname{Lat}(T)$, and $T \in \Phi(X)$. By Proposition $7(1)$, there exists a compact operator $K$ such that $\mathscr{R}(T+K)$ is dense in $X$. Since $T+K \in \Phi(X)$, we have $\mathscr{R}(T+K)=X$. Thus, ind $(T)=\operatorname{ind}(T+K) \geq 0$.

Theorem 9. Assume $\mathscr{E}$ is either an $E U N$ or an $E L N$. Set $A=\operatorname{Alg}(\mathscr{E})$. The following are equivalent for $T \in A$ :

(1) $T \in \Phi^{0}(X)$;

(2) $T$ is invertible in $A$ modulo $\mathscr{F}(A)$;

(3) $\exists F \in \mathscr{F}(A)$ such that $T+F$ is invertible.

Proof. We assume $\mathscr{E}$ is an EUN. The proof when $\mathscr{E}$ is an ELN is similar.

Assume $T \in \Phi^{0}(X)$. By Proposition $7, \exists K \in \overline{\mathscr{F}}(A)$ such that $\mathscr{R}(T+K)$ is dense. Now $T+K \in \Phi^{0}(X)$, so $\mathscr{R}(T+K)=X$ and $\mathscr{N}(T+K)=\{0\}$. It follows that $T+K$ is invertible in $B(X)$, therefore in $A$ [Proposition 2]. Since $K \in \overline{F(A)}$ and the set of invertibles in $A$ is open, $\exists F \in \mathscr{F}(A)$ such that $T+F$ is invertible. This proves (3).

It is clear that (3) implies both (1) and (2). Assume (2) holds, so $\exists S \in A$ and $\exists F, G \in \mathscr{F}(A)$ such that $S T=I-F$ and $T S=I-G$. Now both $S, T \in A$ and $S, T \in \Phi(X)$, so by Corollary 8 ind $(T) \geq 0$ and $\operatorname{ind}(S) \geq 0$. Also, $0=\operatorname{ind}(I-F)=\operatorname{ind}(S T)=\operatorname{ind}(S)+\operatorname{ind}(T)$. Therefore, $\operatorname{ind}(T)=0$, so (1) holds.

For $T \in B(X)$, let $\sigma_{W}(T)$ denote the Weyl spectrum of $T$,

$$
\sigma_{W}(T)=\left\{\lambda \in \mathbf{C}:(\lambda-T) \notin \Phi^{0}(X)\right\} .
$$

It is well known that

$$
\sigma_{W}(T)=\bigcap\{\sigma(T+K): K \in B(X), K \text { compact }\},
$$

[4, Theorem 5.4, p. 180]. 
Corollary 10. Assume $\mathscr{E} \subseteq \operatorname{Lat}(T)$, and $\mathscr{E}$ is either an EUN or an ELN. Then

$$
\sigma_{W}(T)=\bigcap\{\sigma(T+J): J \in \mathscr{F}(A)\} .
$$

Proof. The inclusion $\sigma_{W}(T) \subseteq \bigcap\{\sigma(T+J): J \in \mathscr{F}(A)\}$ follows from the formula above. Now suppose $\lambda \notin \sigma_{W}(T)$, so $\lambda-T \in \Phi^{0}(X)$. Then by Theorem 9, $\exists J \in \mathscr{F}(A)$ such that $(\lambda-T)-J$ is invertible. Thus, $\lambda \notin \sigma(T+J)$. This establishes the reverse inclusion.

\section{Characterization}

In this section we give a characterization in terms of spectral properties of $T$ for when there exists an EUN or an ELN in $\operatorname{Lat}(T)$.

For $\lambda \in \mathbf{C}$ and $m$ a positive integer, let $N(\lambda, m)=\mathscr{N}\left((\lambda-T)^{m}\right)$. Any vector in $N(\lambda, m)$ for some $\lambda$ and $m$ is called a principal vector of $T$, and we let $\mathscr{P}(T)$ denote the set of all principal vectors of $T$.

Theorem 10. Let $X$ be a separable Banach space, and let $T \in B(X)$.

(1) $\exists \mathscr{E}$ an $E U N$ with $\mathscr{E} \subseteq \operatorname{Lat}(T)$ if and only if $X$ is the closed linear span of $\mathscr{P}(T)$.

(2) $\exists \mathscr{E}$ an $E L N$ with $\mathscr{E} \subseteq \operatorname{Lat}(T)$ if and only if the closed linear span of $\mathscr{P}\left(T^{*}\right)$ is a separable total subspace of $X^{*}$.

Proof. First assume $\mathscr{E}$ is an EUN with $\mathscr{E}=\left\{M_{n}: 0 \leq n \leq \infty\right\} \subseteq \operatorname{Lat}(T)$. Now each $M_{n}$ is f.d. and $T\left(M_{n}\right) \subseteq M_{n}$. By [6, pp. 336-338], $M_{n}$ is the span of principal vectors of $T$. But the closed linear span of $\left\{M_{n}: n \geq 0\right\}$ is all of $X$, so the closed linear span of $\mathscr{P}(T)$ is $X$.

Conversely, assume $X$ is the closed linear span of $\mathscr{P}(T)$. For $x \in N(\lambda, m)$, let $M(x)=\operatorname{span}\left\{x, T x, T^{2} x, \ldots, T^{m-1} x\right\}$. We claim that $M(x)$ is $T$ invariant. To prove this it suffices to show that $T^{m} x \in M(x)$. This follows from the equation $0=(\lambda-T)^{m} x=\sum_{k=0}^{m}\left(\begin{array}{l}n \\ k\end{array}\right) \lambda^{k}(-T)^{m-k} x$. Let $X_{0}=\operatorname{span}(\mathscr{P}(T))$. Let $\left\{y_{n}\right\}_{n \geq 1}$ be a countable dense subset of $X_{0}$. Each $y_{n}$ has the form

$$
y_{n}=\sum_{k=1}^{m_{n}} \lambda_{k} x_{n, k}
$$

where each $x_{n, k}$ is a principal vector of $T$. Then

$$
W=\left\{M\left(x_{n, k}\right): 1 \leq k \leq m_{n}, n \geq 1\right\}
$$

is a collection of $T$-invariant f.d. subspaces of $X$, and as $\left\{y_{n}\right\}_{n \geq 1} \subseteq \operatorname{span}(W)$, we have $\operatorname{span}(W)^{-}=X$. Relabel the subspaces in $W$ as $\left\{Y_{n}: n \geq 1\right\}$. Set $M_{0}=\{0\}, M_{n}=\operatorname{span}\left\{Y_{k}: 1 \leq k \leq n\right\}, M_{\infty}=X$. Then $\mathscr{E}=\left\{M_{n}: 0 \leq n \leq\right.$ $\infty\}$ is an EUN and $\mathscr{E} \subseteq \operatorname{Lat}(T)$.

Assume $\mathscr{E}=\left\{M_{n}: 0 \leq n \leq \infty\right\}$ is an ELN with $\mathscr{E} \subseteq \operatorname{Lat}(T)$. Since $M_{n}$ has finite codimension in $X$, it follows that $M_{n}^{\perp}$ is f.d. for $1 \leq n<\infty$. Also, $M_{n}^{\perp}$ is $T^{*}$-invariant for all $n$. Now the closed linear span of $\left\{M_{n}^{\perp}: 1 \leq n<\infty\right\}$ is a separable and total subspace of $X^{*}$. The argument in the proof of (1) shows that $\operatorname{span}\left(\mathscr{P}\left(T^{*}\right)\right)^{-}$contains this subspace.

Conversely, assume $\operatorname{span}\left(\mathscr{P}\left(T^{*}\right)\right)^{-}$is separable and total in $X^{*}$. As in the argument for part (1), $\exists\left\{W_{n}: n \geq 0\right\}$ such that each $W_{n}$ is a f.d., $T^{*}$-invariant subspace, $W_{0}=\{0\}, W_{n} \subseteq W_{n+1}$, and

$$
\operatorname{span}\left(\mathscr{P}\left(T^{*}\right)\right)^{-}=\operatorname{span}\left\{W_{n}: n \geq 1\right\}^{-} .
$$

Let $M_{n}=\left\{x \in X: \alpha(x)=0\right.$ for all $\left.\alpha \in W_{n}\right\}$ for $n \geq 0$. Set $M_{0}=$ $X, M_{\infty}=\{0\}$. It is easy to check that $\mathscr{E}=\left\{M_{n}: 0 \leq n \leq \infty\right\}$ is an ELN with $\mathscr{E} \subseteq \operatorname{Lat}(T)$. 


\section{OPEN PROBLEMS}

When $\mathscr{E}$ is either an EUN or an ELN, and $\mathscr{E} \subseteq \operatorname{Lat}(T)$, then this fact affects the spectral and Fredholm properties of $T$. We believe that a similar situation holds in the case of certain Volterra-type integral operators.

Let $X$ be some Banach space of measurable functions on $[0, \infty), L^{p}[0, \infty)$ for example. Assume that $K(x, t)$ is a measurable function such that the integral operator

$$
V(f)(x)=\int_{0}^{x} K(x, t) f(t) d t \quad(f \in X)
$$

is a bounded operator on $X$. For $a \geq 0$, let

$$
M_{a}=\{f \in X: f \equiv 0 \text { a.e. on }[0, a]\}, \quad M_{\infty}=\{0\} .
$$

Then $\mathfrak{M}=\left\{M_{a}: 0 \leq a \leq \infty\right\}$ is a continuous nest and $\mathfrak{M} \subseteq \operatorname{Lat}(V)$. Two open questions concerning $V$ are:

Question 1. Is $\sigma(V)$ connected?

Question 2. If $\lambda-V \in \Phi(X)$, then is $\operatorname{ind}(\lambda-V) \leq 0$ ?

These questions are considered in Barnes' paper [1].

There are similar open questions concerning the operator

$$
U(f)(x)=\int_{x}^{\infty} K(x, t) f(t) d t \quad(f \in X),
$$

assuming that $U \in B(X)$. Note that $\operatorname{Lat}(U)$ contains the continuous nest $\mathfrak{N}=\left\{N_{a}: 0 \leq a \leq \infty\right\}$, where

$$
N_{a}=\{f \in X: f \equiv 0 \text { a.e. on }[a, \infty)\}, \quad N_{\infty}=X .
$$

Question 3. Is $\sigma(U)$ connected?

Question 4. If $\lambda-U \in \Phi(X)$, then is $\operatorname{ind}(\lambda-U) \geq 0$ ?

\section{REFERENCES}

1. B. Barnes, Spectral properties of linear Volterra operators, J. Operator Theory 24 (1990), 365-382.

2. J. Clauss, Elementary chains of invariant subspaces of a Banach space, Canad. J. Math. (to appear).

3. J. Ringrose, Compact non-self-adjoint operators, Van Nostrand Reinhold, London, 1971.

4. M. Schechter, Principles of functional analysis, Academic Press, New York, 1971.

5. A. Shields, Weighted shift operators and analytic function theory, Topics in Operator Theory, Math. Surveys, no. 13, Amer. Math. Soc., Providence, RI, 1974, pp. 49-128.

6. A. Taylor and D. Lay, Introduction to functional analysis, 2nd ed., Wiley, New York, 1980.

Department of Mathematics, University of Oregon, Eugene, Oregon 97403

Department of Mathematics and Computer Science, Augustana College, Rock ISLAND, ILLINOIS 61201 